\title{
Oral HPV infection in a bone marrow transplantation patient: a case report with atypical clinical presentation and unexpected outcome
}

\begin{abstract}
HPV (Human Papilloma Virus) is one of the most prevalent infections worlwide. Oral HPV infection may be associated with different diseases of oral cavitie. Although oral HPV infection occurs frequently, it rarely causes lesions. An increased rate of oral HPV-induced lesions is observed in people with an impaired immune system. The most common conditions induced by oral HPV infection are focal epithelial hyperplasia, oral condylomas and oral papillomas. We reported a case of oral HPV lesion in a bone marrow transplantation patient with atypical clinical presentation and unexpected outcome.
\end{abstract}

Keywords: HPV, bone marrow transplantation, leukemia.

[Braz J Infect Dis 2010;14(1):89-91] @Elsevier Editora Ltda.

\section{INTRODUCTION}

Human Papilloma Viruses (HPVs) are a large family of DNA viruses capable of infecting a variety of epithelial surfaces, including both mucosal and cutaneous epithelium. HPV is one of the most prevalent infections worldwide with several millions of new cases diagnosed yearly. There are more than $120 \mathrm{HPV}$ types, and at least 30 of these have been detected in the oral cavity. ${ }^{1-3}$

Most often HPV lesions appear on the lips, but they have also been reported on oral mucosa, tongue, gums and palate. At the same time, it should be noted that oral HPV infections are mostly asymptomatic. Although oral HPV-related lesions are rare, they are mostly associated with genital HPV types. ${ }^{3,4}$

An increased rate of oral HPV-induced lesions is observed in people with impaired immune system, especially in HIV-positive individuals. The most common conditions induced by oral HPV infection are focal epithelial hyperplasia, oral condylomas and oral papillomas. Oral HPV infection has been found to be associated with some cases of oropharyngeal cancer, but it is not the main risk factor for this kind of cancer. ${ }^{3,5}$

Oral warts and papillomas in bone marrow transplantation (BMT) patients have been reported 1-3 years after the transplantation. ${ }^{4}$

We reported a case of oral HPV lesion in a BMT patient with atypical clinical presentation and unexpected outcome.

\section{CASE REPORT}

A 41-year-old man, with 5-year-old history of bone marrow transplantation, was referred to the Dental Service of Hematology Center/UNICAMP, on October 2001 for a routine check-up.

The medical history revealed that the patient received the allogeneic bone marrow transplantation (June 1996) from a human leukocyte antigen (HLA)-identical sibling, after a conditioning regimen with cyclophosphamide ( $\mathrm{Cph}$ ) and total body irradiation (TBI). GVHD prophylaxis was performed with cyclosporine A (CsA). On day 200 after the transplantation, following withdrawal of CsA, he presented extensive chronic graft versus host disease (GVHD) on the eyes, skin, lungs and liver. Complete physical examination, pulmonary function tests, complete blood counts, liver function tests, ophthalmologic examination with Schirmer test and biopsies from the face and the trunk
Authors

Claudio Maranhão Pereira $^{1}$, DDS, PhD Laura Guimarães Melo ${ }^{2}$ Maysa Milazzo Correa ${ }^{2}$ Cármino Antônio Souza ${ }^{3}$, $\mathrm{MD}, \mathrm{PhD}$

Maria Elvira P. Corrêa ${ }^{4}$ DDS, PhD

${ }^{1}$ Assistant Professor in Oral Pathology, School of Dentistry, Paulista

University, Goiânia/

GO-Brazil and Assistant

Professor in Pathology,

Estácio de Sá University,

Goiânia/GO-Brazil.

${ }^{2}$ Dentistry student, School of Dentistry, Paulista

University, Goiânia/GO-

Brazil

${ }^{3}$ Assistant Professor in

Hematology Ambulatory,

Hematology and

Hemotherapy Center, State

University of Campinas,

São Paulo, Brazil

${ }^{4}$ Assistant Professor in

Dentistry Ambulatory,

Hematology and

Hemotherapy Center, State

University of Campinas,

São Paulo, Brazil

Submitted on: 09/18/2009 Approved on: 11/19/2009

Correspondence to:

Cláudio Maranhão

Pereira

Faculdade de

Odontologia da

Universidade Paulista,

campus Flamboyant

Patologia Oral

-Diagnóstico Oral

BR-153, KM 503, Fazenda Botafogo - CEP: 74845-

090 - Goiânia/GO - Brazi

$+556232394000$

E-mail:

claudiomaranhao@

hotmail.com;

claudio.maranhao@

go.estacio.br

We declare no conflict of interest. 
supported the diagnosis of chronic GVHD. Treatment with oral prednisone $30 \mathrm{mg} /$ day and CsA $250 \mathrm{mg}$ /day was started, with improvement of the lesions and, the pulmonary and liver function after one month. CsA was tapered gradually until suspension on day 279 after transplantation. Oral examination was performed before the BMT, and he has been followed by the Dental Service of Hematology Center/ UNICAMP since then. The patient had no oral complaints and had only been submitted to periodontal treatment.

However, on October 2001, the oral clinical examination showed a white plaque on the left soft palate, with almost $3.0 \times 3.0 \mathrm{~cm}$ of extension, firm, non removable and asymptomatic (Figure 1). At that time the patient didn't receive GVHD therapy because the disease was under controll, and there were neither quantitative nor qualitative alterations in his immunologic system.

Figure 1: Clinical exam showed the white plaque on the left soft palate of the lesion at the time of the first examination.

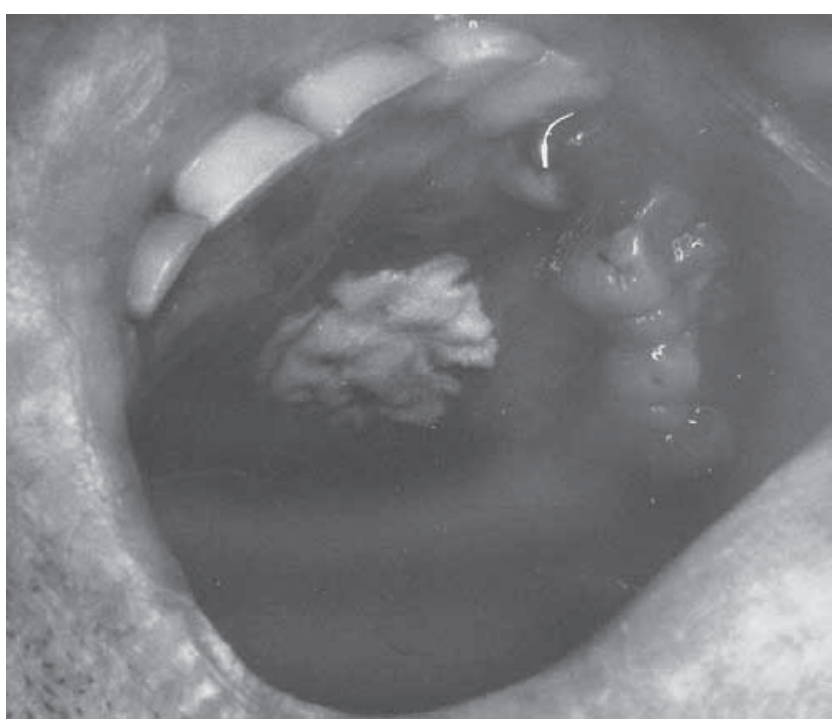

Figure 2: Microscopic exam revealed the hyperkeratosis, acanthosis, epithelium cells with picnotic and hyaline cytoplasm, the presence of vacuolized cells with eosinophilic intercellular inclusions and gross basophilic granules of keratohyaline.

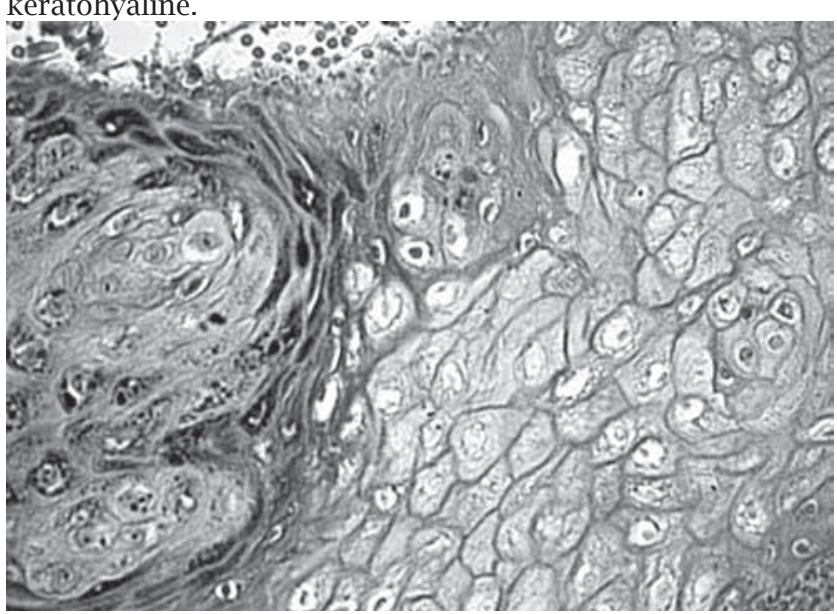

Figure 3: Immunohistochemistry tests concluded the final diagnosis of the HPV infection.

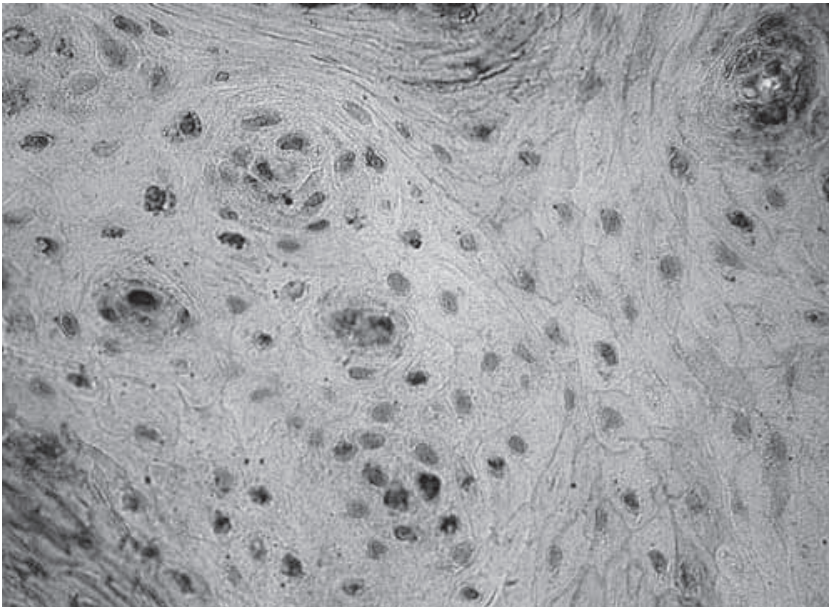

A biopsy of the lesion was performed and the microscopic exam revealed hyperkeratosis, acanthosis, epithelium cells with picnotic and hyaline cytoplasm, the presence of vacuolized cells with eosinophilic intercellular inclusions and gross basophilic granules of keratohyaline, suggesting HPV infection (Figure 2). Data from immunohistochemestry tests confirmed the diagnosis of the HPV infection (Figure 3).

The patient was referred to the Dermatologic Service of Clinic Hospital/UNICAMP in January 2002 and was submitted to Nd:YAG laser for excision of this lesion because of the lower risk of bleeding compared to other surgical techniques. However, the final results were not as expected. The patient returned on April 2002 with a recurrent and exacerbated lesion. Clinic exam revealed the plaque on the left hard and soft palate covering also the alveolar ridges and gingival papilla, spread throughout an area nearly to $6.0 \mathrm{x}$ $5.0 \mathrm{~cm}$ (Figure 4).

Figure 4: Four months after laser therapy, clinic exam revealed the plaque on the left hard and soft palate covering also the alveolar ridges and gingival papilla.

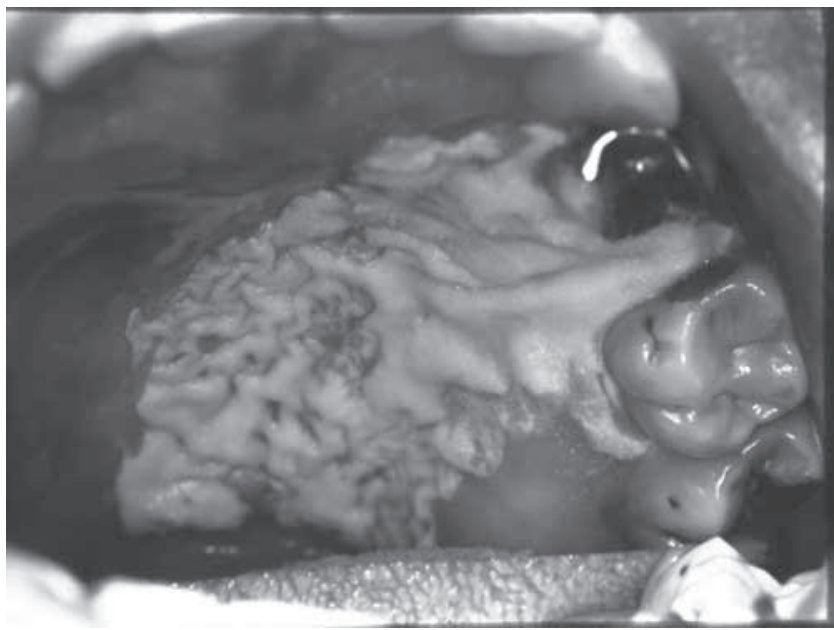


On June 2002 the patient returned for a follow-up visit and the lesion showed a spontaneous partial regression (Figure 5). However, the lesion has not been completely healed so far. The appearance of the white plate on October 2001 and the start of spontaneous regression of it on June 2002 occurred regardless with medical treatment or quantitative and qualitative immunological alterations.

Because the lesion did not present asymptomatic and relevant clinical features, none medication was placed. The patient has been followed and the lesion remained unchanged. The patient has been followed at the Hematology Center/ UNICAMP, and has no medical changing so far

Figure 5: Spontaneous partial regression of the lesion.

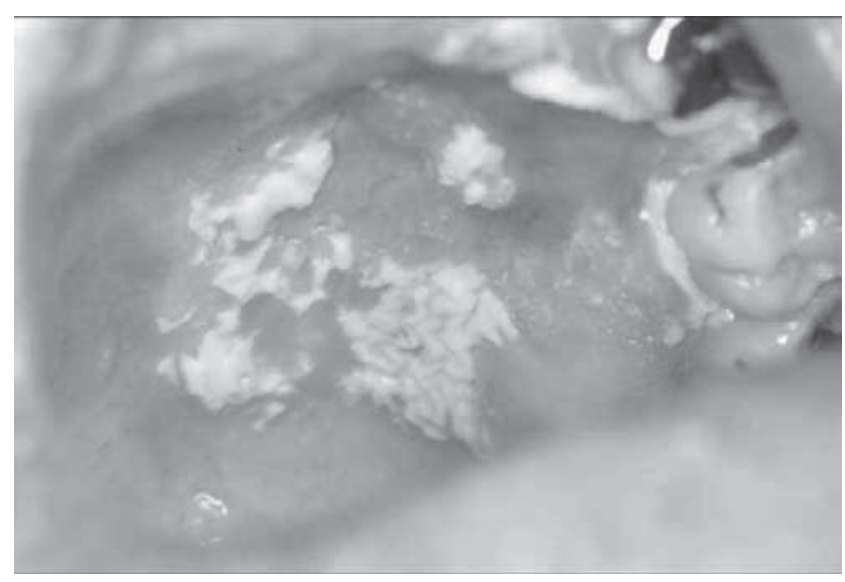

\section{DISCUSSION}

Oral HPV infection may be associated with different diseases of oral cavities, but although this infection occurs frequently, it rarely causes lesions. The gross appearance of oral warts varies greatly and often reflects the specific HPV genotype causing the lesion. Often, the clinical appearance of lesions is reflective of the specific HPV genotype causing the lesion. For instance, HPV genotypes 6 and 11, the most common causes of genital warts, tend to cause condyloma accuminatum in the oral cavity. HPV genotypes 1,2, and 7, which are associated with cutaneous warts, cause firm, sessile, oral common warts. HPV genotypes 13 and 32, which have been described exclusively in the oral cavity, are the cause of oral focal epithelial hyperplasia. ${ }^{6}$

Post-transplantation patients are at increased risk for oral human papillomavirus infections, especially in immunocompromised patients, normally those on high-dose therapy for GVHD. It appears that most cases represent the reactivation of latent virus with patients reporting hand or genital lesions at times prior to transplant. 7,8

BMT complications after 12 months of allogeneic transplants are associated with chronic complications of conditioning regimens, GVHD, and late infections. Infections are infrequent, but an increased incidence of oral HPV infections has been noted in some centers. The presentation of these oral lesions can vary from exophytic oral verrucous vulgaris-like masses to more flat condyloma acuminata-like appearance. ${ }^{4,9,10}$ This case described a leukoplakia-like white plaque on the left soft palate. Its clinical appearance is very uncommon and did not describe in the medical literature.

Treatment must be determined on a case-by-case basis. Many times, the further recovery of immune function will lead to reduction and elimination of oral HPV lesions. On the other hand, it becomes necessary to consider surgical, laser therapy or cryotherapy for the removal of oral HPV lesions, intralesional injections of IFN- $\alpha$ may prove effective for recurrent lesions. ${ }^{4,9,10}$ Herein the case reported was received Nd:YAG laser for excision of this lesion, but the final results were not as expected. After three months the patient returned with a recurrent and exacerbated lesion on the left hard and soft palate covering also the alveolar ridges and gingival papilla, spread to an area around 6.0 $\mathrm{X} 5.0 \mathrm{~cm}$.

However, the lesion started to shrink spontaneously after two months. Since the appearance of the white plate and beginning of the spontaneous regression after nine months, there were no changes in the medical treatment of the patient, as well as no quantitative and qualitative alterations in his immunologic system. Additionally, the lesion has not come to a complete regression until the present moment. No factor could be found, inherent or not to BMT, that could significantly contribute to the spontaneous regression of the lesion in this patient; therefore, it is necessary further studies.

\section{REFERENCES}

1. Llamas-Martínez S, Esparza-Gómez G, Campo-Trapero J et al. Genotypic determination by PCR-RFLP of human papillomavirus in normal oral mucosa, oral leukoplakia and oral squamous cell carcinoma samples in Madrid (Spain). Anticancer Res. 2008; 28(6A):3733-41.

2. Varnai AD, Bollmann M, Bankfalvi A et al. The prevalence and distribution of human papillomavirus genotypes in oral epithelial hyperplasia: proposal of a concept. J Oral Pathol Med. 2009; 38(2):181-7.

3. Baccaglini L, Atkinson JC, Patton LL, Glick M, Ficarra G, Peterson DE. Management of oral lesions in HIV-positive patients. Oral Surg Oral Med Oral Pathol Oral Radiol Endod. 2007; 103 Suppl:S50.e123.

4. Schubert MM. Oral manifestations of viral infections in immunocompromised patients. Curr Opin Dent 1991; 1:384-97.

5. Cianfriglia F, Di Gregorio DA, Cianfriglia C, Marandino F, Perrone Donnorso R, Vocaturo A. Incidence of human papillomavirus infection in oral leukoplakia. Indications for a viral aetiology. J Exp Clin Cancer Res. 2006; 25(1):21-8.

6. Chang F, Syrjanen S, Kellokoski J, Syrjanen K. Human papillomavirus (HPV) infections and their associations with oral disease. J Oral Pathol Med 1991; 20:305-17.

7. Ochs L, Shu XO, Miller J et al. Late infections after allogeneic bone marrow transplantation: Comparison of incidence in related and unrelated donor transplant recipients. Blood 1995; 86:3979-86.

8. Garfunkel AA, Tager N, Chausu S, Chausu G et al. Oral complications in bone marrow transplantation patients: recent advances. Isr J Med Sci 1994; 81:120-4.

9. Kusne S, Krystofiak S. Infection control issues after bone marrow transplantation. Curr Opin Infect Dis 2001; 14:427-31.

10. Offidani M, Corvatta L, Olivieri A et al. Infectious complications after autologous peripheral blood progenitor cell transplantation followed by G-CSF. Bone Marrow Transplant 1999; 24:1079-87. 